\title{
Binary black hole mergers: large kicks for generic spin orientations
}

\author{
Wolfgang Tichy, Pedro Marronetti \\ Department of Physics, Florida Atlantic University, Boca Raton, FL 33431, USA
}

\begin{abstract}
We present results from several simulations of equal mass black holes with spin. The spin magnitudes are $S / m^{2}=0.8$ in all cases, but we vary the spin orientations arbitrarily, in and outside the orbital plane. We find that in all but one case the final merged black hole acquires a kick of more than $1000 \mathrm{~km} / \mathrm{s}$, indicating that kicks of this magnitude are likely to be generic and should be expected for mergers with general spin orientations. The maximum kick velocity we find is $2500 \mathrm{~km} / \mathrm{s}$ and occurs for initial spins which are anti-aligned in the initial orbital plane.
\end{abstract}

PACS numbers: $04.25 . \mathrm{Dm}, 04.30 . \mathrm{Db}, 04.70 . \mathrm{Bw}, 95.30 . \mathrm{Sf}, 97.60 . \mathrm{Lf}$

\section{INTRODUCTION}

Currently several laser-interferometric gravitational wave detectors such as TAMA [1], LIGO [2] and GEO [3] are already operating, while several others are in the planning or construction phase [4]. One of the most promising sources for these detectors are the inspirals and mergers of binary black holes. The gravitational waves radiated during the inspiral and merger of two black holes carry energy, linear momentum and angular momentum fluxes. The radiated energy is positive and thus the system will always lose energy. Except in the case of certain highly symmetric situations, such as head on collisions of equal mass non-spinning black holes, the system usually also loses angular momentum. Generic systems with either unequal masses or spins with no particular alignment will also radiate linear momentum, resulting in a non-zero residual velocity (also known as recoil or "kick") of the final black hole. The magnitude of this recoil is important in a variety of astrophysical scenarios, such as the cosmological evolution of supermassive black holes [5, 6, 7, , 8, 9, 10, 11, 12] or the growth and retention of intermediate-mass black holes in dense stellar clusters [13, 14, 15, 16, 17, 18, 19]. For a binary in almost circular orbit, the direction of the instantaneous linear momentum flux rotates in the orbital plane with the angular velocity of the system. Thus, when the binary goes through one orbital period, the average linear momentum flux will be close to zero. The only net effect comes from the fact that the inspiral orbits are not perfect circles. Most of the kick is thus accumulated during the last orbit and subsequent plunge of the two holes, when the motion of the two holes is no longer quasi-circular and the averaged linear momentum flux is much larger. Several analytical estimates of the kick velocity have been published in recent years [20, 21, 22, 23]. All these estimates have been derived using approximations (such as postNewtonian theory) which break down during the last orbit and the subsequent merger. Hence these calculations contain a high degree of uncertainty. In addition, there are calculations which employ the close limit approximation [24, 25]. However, these calculations can only model well the plunge and miss contributions from the last orbit. Furthermore, results from several numerical simula- tions have been published [26, 27, 28, 29, 30, 31, 32, 33. recently. The resulting maximum kick velocities found so far are: $175 \mathrm{~km} / \mathrm{s}$ for non-spinning unequal mass black holes [28], and $2500 \mathrm{~km} / \mathrm{s}$ for equal mass black holes with anti-aligned spins $S / m^{2} \approx \pm 0.8$ in the orbital plane [31]. The latter value is so high that the final black hole would escape e.g. both from dwarf elliptical and spheroidal galaxies (with typical escapes velocities of below $300 \mathrm{~km} / \mathrm{s}$ ) and from giant elliptical galaxies $(2000 \mathrm{~km} / \mathrm{s})$ [5]. Extrapolations from simulations of black hole binaries with spins in the orbital plane predict velocities as high as $4000 \mathrm{~km} / \mathrm{s}$ 32]. The question thus arises if such high kick velocities are generic for spinning black holes, or if the results of $2500 \mathrm{~km} / \mathrm{s}$ or even $4000 \mathrm{~km} / \mathrm{s}$ arise only if the initial spins are exactly anti-aligned and confined to the initial orbital plane. To answer this question we have performed several simulations of black hole binaries on initially approximately circular orbits with spins in arbitrary directions. We find that except for one case the kick velocities are well above $1000 \mathrm{~km} / \mathrm{s}$. Thus, kicks of this magnitude seem to be a generic feature of black hole mergers with spin around or above $S / m^{2} \approx 0.8$

\section{NUMERICAL TECHNIQUES}

Our simulations are performed using the "moving punctures" method [34, 35] with the BAM code [36, 37] which allows us to use moving nested refinement boxes. We use 10 levels of 2:1 refinements. The outer boundaries are located $240 M$ away from the initial center of mass ( $M$ being the sum of the initial black hole masses), and our resolution ranges between $9 M$ on the outermost box to $M / 56.9$ near the black holes. Since our simulations have no particular symmetries we cannot use the usual memory saving techniques and simulate only one quadrant of the numerical domain. Thus our simulations take four times more memory and time than e.g. the ones reported in 38]. This makes standard convergence tests very costly. Thus, the goal of this work is merely to give kick velocity estimates which can be used in astrophysics, instead of extremely accurate results. For the same reason, we present a convergence test for only one particular 

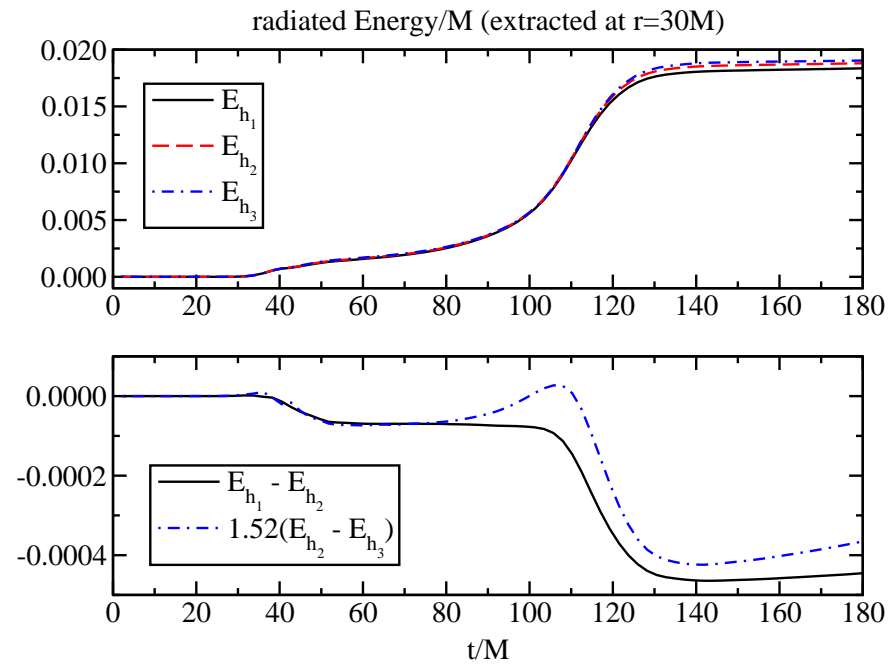

FIG. 1: This plot shows the energy radiated during the merger of a binary where both initial spins $\left(S / \mathrm{m}^{2}=0.8\right)$ are antialigned to the orbital angular momentum. The upper panel shows the energy radiated for the resolutions $h_{1}=M / 56.9$, $h_{2}=M / 61.6, h_{3}=M / 66.4$. In the lower panel we show the differences scaled for fourth order convergence.

case in order to establish that our numerical setup gives results that are in the convergent regime. In Fig. 11 we show the energy radiated during the merger of a binary where both initial spins have magnitude $S / m^{2}=0.8$ and are anti-aligned to the orbital angular momentum. The lower panel of this plot demonstrates that the resolutions $h_{1}, h_{2}$ and $h_{3}$ are in the convergent regime. All other results which are given below, have been obtained using the lowest resolution $h_{1}=M / 56.9$. From this study it is also possible to obtain error bars due to the finite differencing scheme used here. These error bars are of the order of a few percent. Notice however, that there are other sources of errors, the biggest of which is the extraction of the radiated energy and momentum at a finite radius. This radius should ideally be far from the sources. However, the outer regions of our grid are not very well resolved, since the highly refined boxes do not extend far from each black hole. We find that the best compromise for our grid structure is to extract at $r_{e x} \sim 30 M$. Figure 2 shows the radiated momentum in the $z$-direction extracted at different radii. In this example both initial spins are perpendicular to each other and lie in the initial orbital plane. We use the difference between these results at different radii as error bars for the kick velocities presented below.

\section{RESULTS}

In order to gauge possible kick sizes we have performed several simulations with different spin orientations. In each case the spin magnitude of each black hole is $S / m^{2}=0.8$ and the initial orbital angular momentum

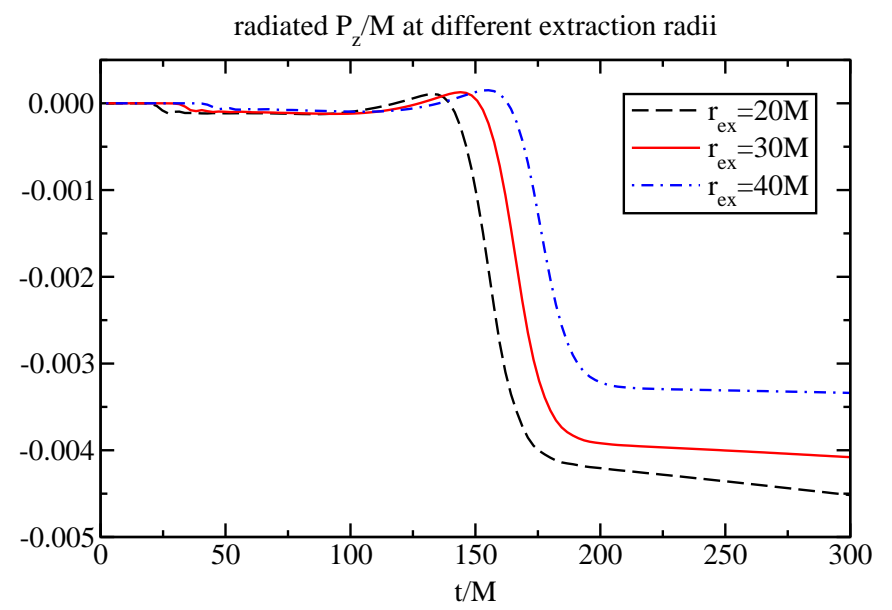

FIG. 2: The z-component of the radiated momentum for the $-1,135 /-1,-135$ run. The initial spins are perpendicular to each other and lie in the initial orbital plane. This plot shows that on our relatively small grids the location of the extraction radius plays a big role and is the main contributor to the error of $\pm 20 \%$ quoted here.

is along the $z$-axis. The magnitude of the orbital angular momentum is chosen such that the orbits are circular according to post-Newtonian approximations [38, 39]. The bare mass parameter $m_{b}$ of each puncture is chosen such that the ADM mass $m$ measured at each puncture is approximately $0.5 M$. Because of these approximations, it is clear that the orbits will have small ellipticities. However, since we are just interested in obtaining a sample of possible kick velocities for different initial conditions such imperfections are not critical.

We label each simulation using seven numbers: $[q, D$, $\left.S_{1} / m^{2}, 90^{\circ}-\theta_{1}, \phi_{1}, S_{2} / m^{2}, 90^{\circ}-\theta_{2}, \phi_{2}\right]$. Defining the larger black hole as \#2, we set $q \equiv m_{2} / m_{1} \geq 1$. D and $S_{A} / m^{2}$ represent the holes' coordinate separation and the spin magnitude of hole $A(A=1,2)$. The angles $\theta_{A}$ and $\phi_{A}$ (given in degrees) determine the direction of each spin by their polar angles with respect to a coordinate system $(\hat{x}, \hat{y}, \hat{z})$, where $\hat{z}$ is the unit vector in the direction of the binary's initial orbital angular momentum, $\hat{x}$ is the unit vector in the direction of the initial linear momentum of the larger hole and $\hat{y}=\hat{z} \wedge \hat{x}$ [42]. Since our runs all have equal masses and spin magnitudes, they are all of the form $\left[1,6 M, 0.8,90^{\circ}-\theta_{1}, \phi_{1}, 0.8,90^{\circ}-\theta_{2}, \phi_{2}\right]$. The runs in Table $\llbracket$ are thus labeled by the angles alone.

From Table I we see that almost all initial spin orientations lead to large kick velocities. The only exception is when both initial black hole spins point in exactly the same direction (see run -31,0/-31,0). In this special case no net linear momentum is radiated (in agreement with post-Newtonian predictions [39]). We find that the largest kick occurs for anti-aligned spins that are perpendicular to the initial angular momentum $J_{\infty}^{A D M}$. However, we also find substantial kicks for other initial spin orientations, and in each case the kick is well above $500 \mathrm{~km} / \mathrm{s}$. This means that kicks of this magni- 


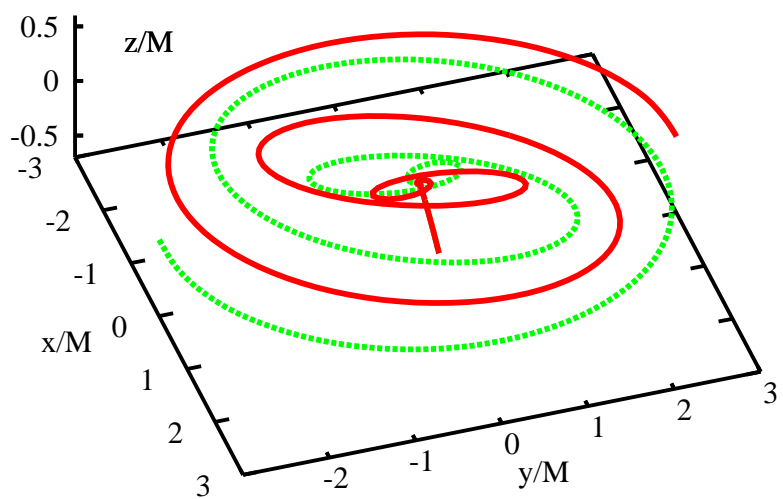

FIG. 3: This plot shows the tracks of the black hole centers for the $-1,180 / 89,180$ run. Here one of the initial spins is in the initial orbital plane, and the other one is perpendicular to the orbital plane. One can see that the orbits do not stay in one plane.

tude should be expected for generic black hole mergers with spins of magnitude of $S / m^{2}=0.8$ or above.

This result can be explained in the following way: it is known that the black hole spins significantly influence the dynamics of the two black holes. For example, the merger can be delayed if both spins are aligned with the

\section{DISCUSSION}

We have performed simulations of equal mass black holes with initial spins of magnitude of $S / m^{2}=0.8$. We have tested several initial spin orientations and find that all but one leads to kick velocities well above $1000 \mathrm{~km} / \mathrm{s}$. Thus kicks between $1000 \mathrm{~km} / \mathrm{s}$ and $2000 \mathrm{~km} / \mathrm{s}$ seem to be a generic feature for spins of magnitude 0.8 with general orientations. Higher spins will lead to even larger kicks. This means that the final black hole will likely escape from dwarf elliptical and spheroidal galaxies (with escape velocities below $300 \mathrm{~km} / \mathrm{s}$ ), and possibly even from giant elliptical galaxies (with escape velocities around $2000 \mathrm{~km} / \mathrm{s}$ ) [5]. This result also has important implications for a variety of astrophysical scenarios, such as the cosmological evolution of supermassive black holes or the growth and retention of intermediate-mass black holes in dense stellar clusters. Note, however, that a more extensive parameter search should be carried out before drawing any definitive conclusions. Furthermore, notice that all our simulations are for the equal mass case. It is clear that the kick velocity will depend on the mass ratio (for two proposed models see [30, 33]), and we expect smaller kicks for the unequal mass case. The probability orbital angular momentum [40]. Or the orbits can be non-planar as in the case of a test mass in orbit around a spinning Kerr black hole [41]. Since the linear momentum radiated depends on the orbit shape, the kick velocity also depends strongly on spin. In fact, if the spins are not parallel to the orbital angular momentum, the spins and the orbital angular momentum both precess so that the resulting orbits do not stay in one plane and become much more complex than the familiar Newtonian circular orbits. Since the instantaneous linear momentum flux changes direction in a less regular way than for circular orbits, one expects that the averaged linear momentum flux is significantly larger than for quasi-circular orbits. As an example of such a more complicated orbit see Fig. 3 which shows the orbits of both black hole centers for the case where one of the spins starts out in the initial orbital plane and the other one perpendicular to this plane. We can clearly see that the orbits do not stay in one plane, and that the kick moves the final merged black hole downward out of the initial orbital plane.

From Figs. 1 and 2 we can see that the bulk of the radiated energy and momentum is emitted within a time of about $40 \mathrm{M}$. This is also the time during which the maximum amplitude of the gravitational waves generated by the merger pass through the detector. I.e. almost the entire kick is accumulated during the final merger phase. Table \gives estimates for the mass and spin of the final black hole.

for the large kicks obtained here might be quite low, if kick velocities drop sufficiently fast for mass ratios different from one.

Almost the entire kick is accumulated during the final merger phase, meaning that small changes in the initial conditions are likely to lead to quite different kick directions and magnitudes. The reason is that a small change in the initial velocities or separation (and also accumulated numerical errors in the orbital phase), will significantly alter the merger time [37], and thus alter the spin orientations just before merger. We therefore expect different trajectories just before the merger which then will lead to different final kicks. Note, however, that this sensitivity to initial conditions does not change our main conclusion that generic spin orientations can lead to very large kicks in the equal mass case.

\section{Acknowledgments}

It is a pleasure to thank B. Brügmann, J. González, M. Hannam, S. Husa, and U. Sperhake for helpful comments and for implementing wave extraction tools in the BAM code. This work was supported by NSF grant PHY0555644. We also acknowledge partial support by the 
Run $\quad 0,90 / 0,-90|-1,135 /-1,-135| 45,0 /-45,180|44,180 /-46,180|-31,0 /-31,0|-1,180 / 89,180|-1,150 / 89,151 \mid-1,120 / 89,119$

\begin{tabular}{c|c|c|c|c|c|ccc}
\hline$M_{\infty}^{\text {ADM }} / M$ & 0.985 & 0.985 & 0.985 & 0.985 & 0.985 & 0.984 & 0.984 & 0.984 \\
$J_{\infty}^{A D M} / M^{2}$ & 0.842 & 0.882 & 0.839 & 0.881 & 0.756 & 1.016 & 1.016 & 1.016 \\
$m / M$ & 0.50 & 0.50 & 0.50 & 0.50 & 0.50 & 0.50 & 0.50 & 0.50 \\
$m_{b} / M$ & 0.30 & 0.30 & 0.30 & 0.30 & 0.30 & 0.30 & 0.30 & 0.30 \\
$D / M$ & 6.000 & 6.000 & 6.000 & 6.000 & 6.000 & 6.000 & 6.000 & 6.000 \\
$P / M$ & 0.140 & 0.140 & 0.140 & 0.140 & 0.147 & 0.133 & 0.133 & 0.133 \\
$L / M^{2}$ & 0.842 & 0.840 & 0.839 & 0.839 & 0.881 & 0.798 & 0.798 & 0.798 \\
$S_{1 x} / m^{2}$ & 0 & -0.566 & 0.566 & -0.576 & 0.684 & -0.800 & -0.693 & -0.400 \\
$S_{1 y} / m^{2}$ & 0.800 & 0.566 & 0 & 0 & 0 & 0 & 0.400 & 0.693 \\
$S_{1 z} / m^{2}$ & 0 & -0.010 & 0.566 & 0.556 & -0.414 & -0.010 & -0.010 & -0.010 \\
$S_{2 x} / m^{2}$ & 0 & -0.566 & -0.566 & -0.556 & 0.684 & -0.010 & -0.009 & -0.005 \\
$S_{2 y} / m^{2}$ & -0.800 & -0.566 & 0 & 0 & 0 & 0 & 0.005 & 0.009 \\
$S_{2 z} / m^{2}$ & 0 & -0.010 & -0.566 & -0.576 & -0.414 & 0.800 & 0.800 & 0.800 \\
\hline$M_{f} / M$ & 0.95 & 0.95 & 0.95 & 0.952 & 0.96 & 0.94 & 0.94 & 0.94 \\
$J_{f} / M_{f}^{2}$ & 0.67 & 0.72 & 0.68 & 0.73 & 0.64 & 0.81 & 0.80 & 0.80 \\
$v(\mathrm{~km} / \mathrm{s})$ & 2500 & 1350 & 580 & 1350 & 0 & 1850 & 2100 & 1950 \\
& \pm 500 & \pm 250 & \pm 150 & \pm 250 & \pm 50 & \pm 350 & \pm 450 & \pm 400
\end{tabular}

TABLE I: Initial parameters and resulting kick velocities. The runs are labeled by the spin orientation angles, which for each hole are the angle between the spin and the initial orbital plane and the angle between the projection of the spin into the orbital plane and the initial linear momentum of hole 2. Here $m_{b}$ is the bare mass parameter of each puncture and $m$ the ADM mass measured at each puncture. The black holes have coordinate separation $D$, with puncture locations $(0, \pm D / 2,0)$ and linear momenta $(\mp P, 0,0)$, so that the initial orbital angular momentum is in the $z$-direction. The spins have a magnitude of $S / m^{2}=0.8$ and point in the various directions listed here, so that the total angular momentum is not necessarily along the $z$-direction. We also list the estimates for the initial ADM mass $M_{\infty}^{A D M}$ and ADM angular momentum $J_{\infty}^{A D M}$. Furthermore, we give the final black hole mass $M_{f}$ and angular momentum $J_{f}$, as well as the kick velocities $v$ for the different cases.

National Computational Science Alliance under Grants PHY060021P and PHY060040T. Simulations were also performed at the Charles E. Schmidt College of Science computer cluster Boca 5.
[1] TAMA, http://tamago.mtk.nao.ac.jp/.

[2] LIGO, http://www.ligo.caltech.edu/.

[3] GEO, http://www.geo600.uni-hannover.de/.

[4] B. Schutz, Class. Quantum Grav. 16, A131 (1999).

[5] D. Merritt, M. Milosavljevic, M. Favata, S. A. Hughes, and D. E. Holz, Astrophys. J. 607, L9 (2004), astro$\mathrm{ph} / 0402057$.

[6] M. Boylan-Kolchin, C.-P. Ma, and E. Quataert, Astrophys. J. 613, L37 (2004), astro-ph/0407488.

[7] Z. Haiman, Astrophys. J. 613, 36 (2004), astro$\mathrm{ph} / 0404196$.

[8] P. Madau and E. Quataert, Astrophys. J. 606, L17 (2004), astro-ph/0403295.

[9] J. Yoo and J. Miralda-Escude, Astrophys. J. 614, L25 (2004), astro-ph/0406217.

[10] M. Volonteri and R. Perna, Mon. Not. Roy. Astron. Soc. 358, 913 (2005), astro-ph/0501345.

[11] N. I. Libeskind, S. Cole, C. S. Frenk, and J. C. Helly, Mon. Not. Roy. Astron. Soc. 368, 1381 (2006), astro$\mathrm{ph} / 0512073$.

[12] M. Micic, T. Abel, and S. Sigurdsson (2005), astro$\mathrm{ph} / 0512123$.

[13] M. C. Miller and D. P. Hamilton, MNRAS 330, 232 (2002).

[14] M. C. Miller and D. P. Hamilton, Astrophysical Journal 576, 894 (2002).

[15] H. Mouri and Y. Taniguchi, Astrophysical Journal 566, L17 (2002).
[16] H. Mouri and Y. Taniguchi, Astrophysical Journal 580, 844 (2002).

[17] K. Gultekin, M. C. Miller, and D. P. Hamilton, Astrophys. J. 616, 221 (2004), astro-ph/0402532.

[18] K. Gultekin, M. Coleman Miller, and D. P. Hamilton, Astrophys. J. 640, 156 (2006), astro-ph/0509885.

[19] R. M. O'Leary, F. A. Rasio, J. M. Fregeau, N. Ivanova, and R. O'Shaughnessy, Astrophys. J. 637, 937 (2006), astro-ph/0508224.

[20] A. G. Wiseman, Phys. Rev. D 46, 1517 (1992).

[21] M. Favata, S. A. Hughes, and D. E. Holz, Astrophys. J. 607, L5 (2004), astro-ph/0402056.

[22] L. Blanchet, M. S. S. Qusailah, and C. M. Will, Astrophys. J. 635, 508 (2005), astro-ph/0507692.

[23] T. Damour and A. Gopakumar, Phys. Rev. D73, 124006 (2006), gr-qc/0602117.

[24] C. F. Sopuerta, N. Yunes, and P. Laguna, Phys. Rev. D74, 124010 (2006), astro-ph/0608600.

[25] C. F. Sopuerta, N. Yunes, and P. Laguna, Astrophys. J. 656, L9 (2007), astro-ph/0611110.

[26] F. Herrmann, D. Shoemaker, and P. Laguna (2006), grqc/0601026.

[27] J. G. Baker et al., Astrophys. J. 653, L93 (2006), astroph/0603204.

[28] J. A. Gonzalez, U. Sperhake, B. Bruegmann, M. Hannam, and S. Husa (2006), gr-qc/0610154.

[29] F. Herrmann, I. Hinder, D. Shoemaker, P. Laguna, and R. A. Matzner (2007), gr-qc/0701143. 
[30] M. Campanelli, C. O. Lousto, Y. Zlochower, and D. Merritt (2007), gr-qc/0701164.

[31] J. A. Gonzalez, M. D. Hannam, U. Sperhake, B. Brugmann, and S. Husa (2007), gr-qc/0702052.

[32] M. Campanelli, C. O. Lousto, Y. Zlochower, and D. Merritt (2007), gr-qc/0702133.

[33] J. G. Baker et al. (2007), astro-ph/0702390.

[34] M. Campanelli, C. O. Lousto, P. Marronetti, and Y. Zlochower, Phys. Rev. Lett. 96, 111101 (2006), grqc/0511048.

[35] J. G. Baker, J. Centrella, D.-I. Choi, M. Koppitz, and J. van Meter, Phys. Rev. Lett. 96, 111102 (2006), grqc/0511103.

[36] B. Bruegmann, W. Tichy, and N. Jansen, Phys. Rev. Lett. 92, 211101 (2004), gr-qc/0312112.
[37] B. Bruegmann, J. Gonzalez, M. Hannam, S. Husa, U. Sperhake, and W. Tichy (2006), gr-qc/0610128.

[38] P. Marronetti, W. Tichy, B. Bruegmann, J. Gonzalez, M. Hannam, S. Husa, and U. Sperhake (2007), grqc/0701123.

[39] L. E. Kidder, Phys. Rev. D52, 821 (1995), grqc/9506022.

[40] M. Campanelli, C. O. Lousto, and Y. Zlochower, Phys. Rev. D74, 041501 (2006), gr-qc/0604012.

[41] M. Campanelli, C. O. Lousto, Y. Zlochower, B. Krishnan, and D. Merritt (2006), gr-qc/0612076.

[42] In this paper, we constructed our initial data sets such that the numerical grid coordinate system $(x, y, z)$ coincides with $(\hat{x}, \hat{y}, \hat{z})$ 